(pepF- $32 \mathrm{aHC}$ ) solely on the basis that TFL-006 displayed lower MFI than HC-10 should be considered with caution.

Second, the "alkali-treatment" of LSAB described by the authors is performed with concentrated guanidinium chloride, a chaotropic agent, and is much harsher than a denaturation induced by a mild $\mathrm{pH}$ change. After this treatment, the MFI of TFL-006 became higher than with acid-treated LSAB, but remained lower than HC-10 MFI that did not reach the same level observed on acid-treated beads. These results indicate that TFL-006 could recognize an isoform of $\beta 2 \mathrm{fHC}$ that is not denatured the same way as the one present on LSAB, and underline that the kind of LSAB denaturing treatment could greatly impact the denaturation process in ways that are not understood yet.

Finally, the authors proposed that our previous results showing that anti-dHLA can still stain iBeads corresponded to antibodies directed against pepF- $32 \mathrm{aHC}{ }^{3,5}$ To us, this is highly unlikely because acid-induced denaturation is known to release $\beta 2 \mathrm{~m}$ from HLA molecules, leading to an almost complete loss of $\beta 2 \mathrm{aHC}$. Moreover, we clearly demonstrated that reactivities resembling those of anti-dHLA can correspond to anti-native HLA (anti-nHLA) antibodies recognizing peculiar epitopes displayed by pepA- $\beta 2 \mathrm{aHC}$ that are conserved on $\beta 2 \mathrm{fHC}$, and/or mixtures of anti-nHLA and anti-dHLA antibodies recognizing the same antigens. ${ }^{4}$
In conclusion, Jucaud et $\mathrm{al}^{1}$ brought additional data about denatured HLA-I molecules borne by LSAB. Their results underline the difficulty of correctly interpreting anti-dHLA patterns using strategies relying on bead-specific cutoffs based on monoclonal antibodies reactivity or chemical treatments of LSAB. As suggested by the authors, the transplant community could plead in favor of the resurgence of iBeads production because this assay proved efficient in eliminating the anti-dHLA detection and showed promising results. ${ }^{2,3,5}$

\section{REFERENCES}

1. Jucaud V, Ravindranath MH, Terasaki PI. Conformational variants of the individual HLA-I antigens on Luminex single antigen beads used in monitoring HLA antibodies: problems and solutions. Transplantation. 2016 doi: 10. 1097/TP.0000000000001420.

2. Otten HG, Verhaar MC, Borst HP, et al. The significance of pretransplant donor-specific antibodies reactive with intact or denatured human leucocyte antigen in kidney transplantation. Clin Exp Immunol. 2013;173:536-543.

3. Visentin J, Guidicelli G, Nong T, et al. Evaluation of the iBeads assay as a tool for identifying class I HLA antibodies. Hum Immunol. 2015;76: $651-656$

4. Visentin J, Guidicelli G, Moreau JF, et al. Deciphering allogeneic antibody response against native and denatured HLA epitopes in organ transplantation. Eur J Immunol. 2015;45:2111-2121.

5. Visentin J, Marroc M, Guidicelli G, et al. Clinical impact of preformed donorspecific denatured class I HLA antibodies after kidney transplantation. Clin Transplant. 2015;29:393-402.

\title{
The Authors' Reply
}

\author{
Mepur H. Ravindranath, MSc, $\mathrm{PhD}^{1}$ and Vadim Jucaud, BSc ${ }^{1}$
}

W e thank Visentin and coauthors ${ }^{1}$ for pointing out that our findings ${ }^{2}$ are in line with their earlier observations. Because the current clinical objective is to detect the antibodies (Abs) binding to peptide-associated $\beta 2$-microglobulinassociated HLA-I heavy chains (pepA- $\beta 2 \mathrm{aHC}$ ), the native HLA-I conformation expressed on the surface of resting cells, the presence of other conformational variants on single antigen beads $(\mathrm{SAB})$ prevents the distinction between clinically relevant and irrelevant HLA Abs, in pre- and posttransplant patients, when the mean fluorescence intensities is below a certain threshold depending on the specific bead. The indiscriminate usage of the term anti-denatured (dn) HLA Abs adds additional concern. El-Awar et $\mathrm{al}^{3}$ have referred peptide-free $\beta 2$-microglobulin-free HLA-I heavy chains (pepF- $\beta 2 \mathrm{fHC}$ ) as natural antibodies or "anti-dnHLA Abs." To distinguish pepA- $\beta 2 \mathrm{aHC}$ from pepF- $\beta 2 \mathrm{fHC}$, the

Received 28 November 2016. Revision received 7 December 2016.

Accepted 11 December 2016.

${ }^{1}$ Terasaki Foundation Laboratory, Los Angeles, CA 90064.

The authors declare no funding or conflicts of interest.

Both authors have equal contribution in formulating, executing the experiments and writing the article.

Correspondence: Mepur H. Ravindranath, MSc, PhD, Terasaki Foundation Laboratory, Los Angeles, CA 90064, USA. (ravimh@terasakilab.org).

Copyright $\odot 2017$ Wolters Kluwer Health, Inc. All rights reserved.

ISSN: 0041-1337/17/10104-0154

DOI: 10.1097/TP.0000000000001629 regular beads are subjected to acid or alkali or heat treatments, which not only releases $\beta 2$-microglobulin and a peptide from the heavy chain (HC) but also alters the physical configuration of the HC, depending on the treatments. Failure to recognize this alteration has created ambiguity and misperception, as in a recent publication. ${ }^{4}$ Opposite conclusions were inferred from the impact of anti-dnHLA Abs using different denaturation methods. Visentin et $\mathrm{al}^{5}$ have used "acid-treated beads" to conclude that anti-dnHLA Abs do not impair graft survival, ${ }^{1}$ whereas Cai et $\mathrm{al}^{6}$ used the "heat-treated $\left(90^{\circ} \mathrm{C}\right)$ beads" to state that "complement-fixing antibodies against denatured HLA and MICA antigens are associated with antibody mediated rejection." The $\beta 2 \mathrm{fHC}$ are also expressed naturally ("open conformers"7) on activated lymphocytes, ${ }^{8}$ and serve as ligands for leukocyte receptors. ${ }^{9}$ Therefore, the naturally occurring (or expressed) $\beta 2 \mathrm{fHC}$ cannot be referred to as dnHLA. Perhaps beads with only $\beta 2 \mathrm{fHC}$ may specifically identify clinically relevant $\beta 2 \mathrm{fHC}$ Abs.

It is true that monoclonal antibodies TFL-006 provided lower mean fluorescence intensities than monoclonal antibodies HC-10 on acid-treated beads (displaying only pepF- $\beta 2 \mathrm{fHC}$ ). Obviously, the acid denaturation (acid-dn) affected the epitope recognition by TFL-006 more strongly than the one recognized by HC-10. The following reasons support the above contention:

- TFL-006, raised by immunizing the folded $\beta 2 \mathrm{~m}$-free HLA-E $\mathrm{E}^{\mathrm{R} 107}$ $\mathrm{HC},{ }^{10}$ recognizes a conformational epitope on $\beta 2 \mathrm{fHC}$. In 
contrast, HC-10 was raised against denatured papainfragments of HLA-B7 and HLA-B40 HC. ${ }^{11}$ Therefore, the TFL-006 epitope may be more sensitive to the acid-dn of SAB compared to the HC-10 epitope.

- Although the TFL-006 epitope $\left({ }^{117}\right.$ AYDGKDY $\left.{ }^{123}\right)$ is located in the $\alpha 2$-domain of $\mathrm{HC},{ }^{12}$ the antigenic determinant $\left(\mathrm{R}^{62}\right)$ of HC-10 is located in the $\alpha 1$-domain. Upon acid, alkali and heat denaturation of $\mathrm{HC}$, the $\alpha 1$-domain is most susceptible to structural changes, because the $\alpha 2$ - and $\alpha 3$-domains contain disulfide bonds. Such denaturation of the $\alpha 1$-domain does not abolish HC-10 reactivity, as Perosa et $\mathrm{al}^{13}$ documented that HC-10 recognizes a linear epitope, but the extreme denaturation of the $\alpha 1$-domain may hinder the accessibility of TFL-006 to its epitope on $\alpha 2$.

Moreover, the epitope of TFL-006 is shared by HLAA/-HLA-B/HLA-Cw/HLA-E/HLA-F and HLA-G, ${ }^{12}$ and the location of the sequence (shown in figures ${ }^{14}$ ) is cryptic in $\beta 2 \mathrm{aHC}$ because $\beta 2$-microglobulin masks the epitope; TFL-006 recognizes only the $\beta 2 \mathrm{fHC} \mathrm{HLA-I.} \mathrm{On} \mathrm{the} \mathrm{other}$ hand, the crypticity of the HC-10 epitope is only dependent on the presence of a peptide in the groove, and the epitope is not shared by all HLA-I antigens coated on SAB. HC-10 recognizes both peptide-free $\beta 2$-microglobulin-associated HLA-I heavy chains (pepF- $\beta 2 \mathrm{aHC}$ ) and $\beta 2 \mathrm{fHC}$.

Visentin et $\mathrm{al}^{5}$ described patients' sera which contain antidnHLA directed against all the beads which are not recognized by TFL-006; however, the HLA-I specificity is not explicit. Nonetheless, we inferred that "anti-dHLA" that can stain iBeads corresponded to antibodies directed against pepF- $\beta 2$ aHC because W6/32 (which confirms the presence of pepA- $\beta 2 \mathrm{aHC}$ and pepF- $\beta 2 \mathrm{aHC}$ ) and HC-10 (which confirms the presence of pepF- $\beta 2 \mathrm{aHC}$ and $\beta 2 \mathrm{fHC}$ ) bind but TFL-006 (confirming the absence of $\beta 2 \mathrm{fHC}$ ) does not. By the process of elimination, only pepA- $\beta 2 \mathrm{aHC}$ and pepF$\beta 2 \mathrm{aHC}$ are present on iBeads. In addition, such "anti-dHLA that can stain iBeads" but are not associated with positive FCXM could be similar to HC-10. Therefore, we concluded that "anti-dHLA that can stain iBeads" mimic HC-10 reactivity in SAB and FXCM. We are aware that some peculiar epitopes can be conserved on all HLA-I conformational variants, but it needs verification as to whether Abs against these epitopes are positive with FXCM.

Finally, we conclude that (i) the manufacturing process of SAB produces a highly heterogeneous product, with varying densities (or concentrations) of the different variants, which impedes recognition of the conformational specificity of
anti-HLA Abs; (ii) there is a need for the resurgence of iBeads to detect HLA Abs specifically targeting pepA- $\beta 2 \mathrm{aHC}$; (iii) a consensus is needed for the terminology used to describe anti-HLA Abs against different conformational variants and (iv) the term "anti-dnHLA Abs" should only be restricted to Abs tested on physicochemically treated SABs, and the name of the treatment should be specified (ie', anti-acid-dnHLA Ab).

\section{REFERENCES}

1. Visentin J, Guidicelli G, Taupin JL. Conformational variants of HLAantigens on Luminex Single Antigen Beads. Transplantation. 2016; 101:e153.

2. Jucaud V, Ravindranath $\mathrm{MH}$, Terasaki PI. Conformational variants of the individual HLA-I antigens on Luminex single antigen beads used in monitoring HLA antibodies: problems and solutions. Transplantation. 2016;101:764-777.

3. El-Awar N, Terasaki P, Hajeer A, et al. 12-P: A Novel HLA class I single antigen bead preparation eliminates false positive reactions attributed to natural antibodies - in the sera of normal males and pre-transplant patients. Human Immunol. 2010;71:S26.

4. Michel K, Santella R, Steers J, et al. Many de novo donor-specific antibodies recognize $\beta 2$-microglobulin-free, but not intact HLA heterodimers. HLA. 2016;87:356-366.

5. Visentin J, Marroc M, Guidicelli G, et al. Clinical impact of preformed donor-specific denatured class I HLA antibodies after kidney transplantation. Clin Transplant. 2015;29:393-402.

6. Cai J, Terasaki PI, Zhu D, et al. Complement-fixing antibodies against denatured HLA and MICA antigens are associated with antibody mediated rejection. Exp Mol Pathol. 2016;100:45-50.

7. Arosa FA, Santos SG, Powis SJ. Open conformers: the hidden face of MHC-I molecules. Trends Immunol. 2007;28:115-23.

8. Pickl WF, Holter W, Stöckl J, et al. Expression of beta 2-microglobulinfree HLA class I alpha-chains on activated T cells requires internalization of HLA class I heterodimers. Immunology. 1996;88:104-9.

9. Allen RL, Trowsdale J. Recognition of classical and heavy chain forms of HLA-B27 by leukocyte receptors. Curr Mol Med. 2004;4:59-65.

10. Ravindranath $\mathrm{MH}$, Terasaki PI, Pham T, et al. Therapeutic preparations of IVIg contain naturally occurring anti-HLA-E antibodies that react with HLA-la (HLA-A/-B/-Cw) alleles. Blood. 2013;121:2013-2028.

11. Stam NJ, Spits H, Ploegh HL. Monoclonal antibodies raised against denatured HLA-B locus heavy chains permit biochemical characterization of certain HLA-C locus products. J Immunol. 1986;137:2299.

12. Ravindranath MH, Zhu D, Pham T, et al. Anti-HLA-E monoclonal antibodies reacting with HLA-la and Ib alleles like IVIg as potential IVIgimmunomimetics: an evolving therapeutic concept. Clin Transpl. 2013:293-305.

13. Perosa F, Luccarelli G, Prete M, et al. Beta 2-microglobulin-free HLA class I heavy chain epitope mimicry by monoclonal antibody HC-10-specific peptide. J Immunol. 2003;171:1918-26.

14. Ravindranath $\mathrm{MH}$, Kaneku H, El-Awar N, et al. Antibodies to HLA-E in nonalloimmunized males: pattern of HLA-la reactivity of anti-HLA-Epositive sera. J Immunol. 2010;185:1935-48. 